\title{
Asteroseismic diagrams for solar-type stars ${ }^{\star}$
}

\author{
A. Mazumdar ${ }^{1,2}$ \\ 1 Instituut voor Sterrenkunde, KU Leuven, Celestijnenlaan 200B, 3001 Leuven, Belgium \\ e-mail: anwesh.mazumdar@ster.kuleuven.ac.be \\ 2 Observatoire de Paris, LESIA, CNRS UMR 8109, 92195 Meudon, France
}

Received 9 November 2004 / Accepted 18 June 2005

\section{ABSTRACT}

We explore the feasibility of applying the Christensen-Dalsgaard diagram to real asteroseismic data and provide quantitative measures of the uncertainty associated with the results. We also propose a new kind of seismic diagram, based on the determination of the locations of sharp acoustic features inside a star. We show that by combining the information about the position of the base of the convective envelope or the He II ionisation zone with a measure of the average large separation, it is possible to constrain the unknown chemical composition or the various parameters characterising the physical processes in the stellar interior. We demonstrate the application of this technique to the analysis of mock data for a CoRoT target star.

Key words. stars: oscillations - stars: interiors

\section{Introduction}

Seismic observations of distant stars, either from the ground or space, is likely to permit the detection of only low degree modes of oscillations $(\ell=0,1,2,3)$. In this context, it is necessary to develop or adapt the existing seismic tools of analysis to extract the maximum amount of information contained in these modes. One approach is through the construction of suitable asteroseismic diagrams - the technique of combining useful features of the frequency spectrum of stars to extract information about the physical processes in the stellar interior that affect the frequencies. The basic idea behind such diagrams is that they are to be constructed in terms of asteroseismic observables and must reveal some structural or evolutionary feature of a star through a calibration of theoretical stellar models.

The most well-known asteroseismic diagram is the socalled Christensen-Dalsgaard diagram, or the C-D diagram, in short (Christensen-Dalsgaard 1988). This diagram exploits the fact that while the average large separation of radial modes, $\left\langle\Delta v_{0}\right\rangle \equiv\left\langle\Delta v_{0}(n)\right\rangle=\langle v(n, 0)-v(n-1,0)\rangle$ reflects the gross properties of a star, like its mass and radius, the average small separation, $\left\langle d_{02}\right\rangle \equiv\left\langle d_{02}(n)\right\rangle=\langle v(n, 0)-v(n-1,2)\rangle$ is more sensitive to the innermost layers of the star, and therefore to its evolutionary state. The averages are computed over several high-order modes (the so-called asymptotic range). By determining the position of a star on this diagram, through measurement of the large and small separations of its oscillation frequencies, it is possible to determine its mass and age.

\footnotetext{
* Section 6 is only available in electronic form at
} http://www. edpsciences.org
In this work, we study the feasibility of using the C-D diagram for real asteroseismic data containing finite errors in the frequencies. We investigate the accuracy to which the mass and age of a star with unknown characteristics (such as the chemical composition or the extent of convective overshoot) can be predicted from the C-D diagram (Sect. 2).

The rest of the paper introduces a new kind of asteroseismic diagram. The acoustic location of sharp features inside a star such as the boundaries of convective zones or ionisation zones can be determined using the oscillatory signal in the frequencies that they produce (e.g., Monteiro et al. 1994; Basu et al. 1994; Roxburgh \& Vorontsov 1994). Considering that the location of these regions cannot be independent of the general stratification inside a star, in Sect. 3 we propose a new diagram, connecting these independent seismic observables and the mean large separation. An application of this seismic diagram to the analysis of synthetic data for the star HD 49933 illustrates how it could help substantially in constructing a reliable model of the star (Sect. 4).

This work utilises several grids of stellar models with different stellar parameters which were constructed using the CESAM evolutionary code (Morel 1997). These models used the OPAL equation of state (Rogers \& Nayfonov 2002) and OPAL opacity tables (Iglesias \& Rogers 1996), complemented by the low-temperature opacity tables of Alexander \& Ferguson (1994). Convection was described by the standard mixing length theory (Henyey et al. 1965) and nuclear reaction rates were obtained from the NACRE compilation (Angulo et al. 1999). Diffusion of helium and heavy elements was not 
considered for the present study. While it is generally accepted that some form of diffusive phenomenon is very likely to exist in stellar interiors, we believe that its inclusion would not alter the results of this work in a qualitative way. The effect of incorporating diffusion would be akin to exploring another dimension in stellar parameter space - the various calibration curves might change in absolute terms, but that would not affect the general conclusions drawn from them.

The p-mode oscillation frequencies of low degree modes $(\ell=0,1,2,3)$ were computed for each model under the adiabatic approximation, using the Aarhus pulsation package, ADIPLS (Christensen-Dalsgaard \& Berthomieu 1991). Throughout the work, we have used these low degree modes which are the only ones relevant for real asteroseismic data.

\section{Sensitivity of the C-D diagram to stellar parameters}

The general idea of the C-D diagram has already been presented in Sect. 1. Recently, Roxburgh \& Vorontsov (2003) have demonstrated that while the frequency separations themselves are affected by the effects of the surface layers, the ratio of the small separation to the large separation, defined as $\left\langle r_{02}\right\rangle \equiv\left\langle r_{02}(n)\right\rangle=\left\langle\frac{d_{02}(n)}{\Delta v_{1}(n)}\right\rangle$, can exclude such effects to a great extent. Similar C-D diagrams can be constructed using these ratios, instead of the small separations themselves (Floranes et al. 2005). A comparison of the classical C-D diagram and its variation for $\ell=0,1$ modes (Mazumdar \& Roxburgh 2003), with small separations as well as the ratio of separations, can be found in the online appendix.

To investigate whether the C-D diagram can indeed be used for real asteroseismic data, we compare the sensitivity of the tracks on the C-D diagram toward different stellar parameters to the uncertainty associated with placing an observed star on this diagram. We estimate the errors in $\left\langle\Delta v_{0}\right\rangle$ and $\left\langle d_{02}\right\rangle$ through a Monte Carlo simulation where random errors with a specified standard deviation $\left(\sigma_{v}=10^{-4} v\right)$ are added to the exact frequencies before computing the separations. Such an error margin in the frequencies is consistent with the expectations from the current and future asteroseismic space missions, MOST (Walker et al. 2003) and CoRoT (Baglin 2003), as well as recent ground-based seismic observations (e.g., Bedding \& Kjeldsen 2003). The variance in the average values of the large and small separations are estimated to be the uncertainties in these quantities for a real data set with comparable errors in frequencies. We find that the error in determining the average large separation is only $\sim 0.02 \mu \mathrm{Hz}$. Thus, although the individual frequency values might have errors up to $\sim 0.3 \mu \mathrm{Hz}$, the average value, $\left\langle\Delta v_{0}\right\rangle$ over a suitably chosen range of radial orders is quite a robust quantity. On the other hand, the uncertainty in the average small separation, $\left\langle d_{02}\right\rangle$ is $\sim 0.0015 \mu \mathrm{Hz}$. Very similar estimates for these uncertainties were found by Audard et al. (1995). However, the errors in determining these quantities would be affected by the total number of modes observed as well as the mass and age of the star. At late stages of evolution, particularly in more massive stars, the presence of mixed non-radial modes reduces the number of modes available for such asymptotic analysis. Typically, this causes an increase in

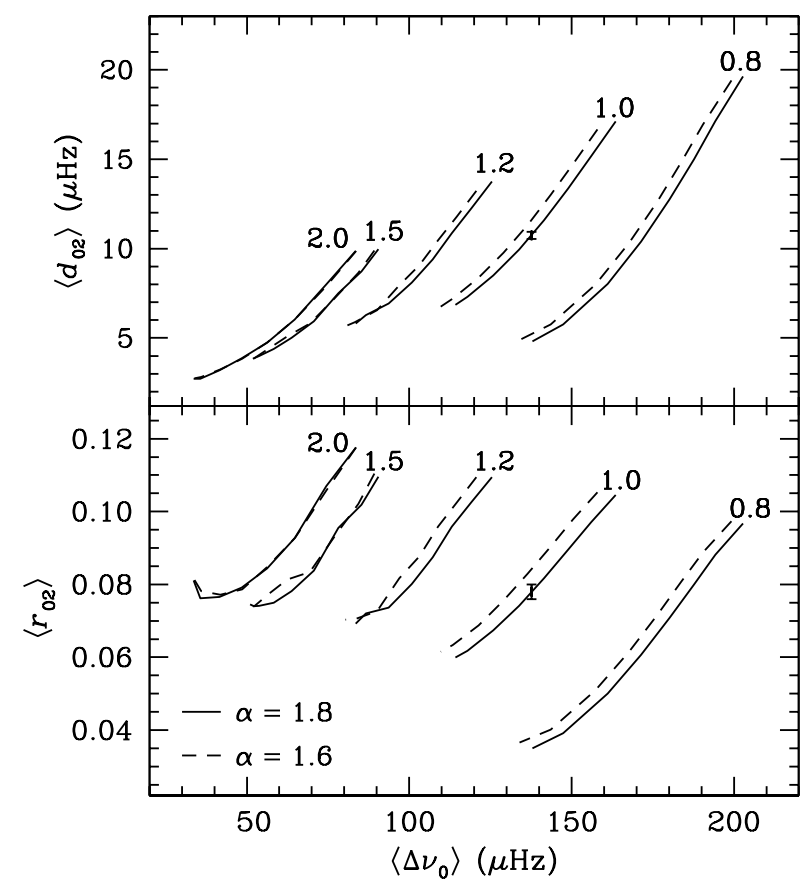

Fig. 1. The variation of the C-D diagram using the average small separations, $\left\langle d_{02}\right\rangle$ (top panel), and the average ratios, $\left\langle r_{02}\right\rangle$ (bottom panel) with the choice of the mixing length parameter, $\alpha$, is shown. Two sets of models have been used - one with $\alpha=1.8$ (solid curves) and another with $\alpha=1.6$ (dashed curves) to illustrate the shift in the tracks. All other stellar parameters are identical in the two sets of models. Representative errorbars corresponding to relative frequency errors of $10^{-4}$ are shown.

the error on the average small separations. The error estimates quoted here, and shown in Figs. 1 to 3 are, therefore, only representative.

The theoretical C-D diagram is typically based on stellar models constructed with a particular choice of parameters, principal among which are the mixing length $\alpha$, the extent of convective core overshoot, $d_{\mathrm{ov}}$ (both $\alpha$ and $d_{\mathrm{ov}}$ being measured in terms of local pressure scale height) and the initial chemical composition (characterised by $\left(X_{0}, Z_{0}\right)$ ). In order to investigate the effect of these parameters on the C-D diagram, we constructed separate grids of stellar models with different values for these parameters. For each of these sets of models, only one parameter (e.g., $\alpha$ ) is varied at a time, keeping the others (viz., $d_{\text {ov }}, X_{0}, Z_{0}$ ) identical, so that each effect can be separately understood. The frequency range for determining the asymptotic average values of the separations is chosen in terms of the scaled frequency, $v / \sqrt{\left(M / M_{\odot}\right) /\left(R / R_{\odot}\right)^{3}}$, where $M$ and $R$ are the mass and radius for each model respectively, to be $1.5 \mathrm{mHz}$ to $3.5 \mathrm{mHz}$. This typically corresponds to averaging over $15 \mathrm{ra}-$ dial orders.

In Fig. 1, we show the variation in the C-D diagrams of $\left\langle d_{02}\right\rangle$ and $\left\langle r_{02}\right\rangle$ with the mixing length parameter, $\alpha$. Each curve on this diagram (and Figs. 2 and 3 ) is an evolutionary track of a given mass, indicated in solar units at the ZAMS end of the track. We consider two sets of models, corresponding to $\alpha=1.8$ and 1.6. Since the outer convective envelope grows thinner for higher masses, naturally, the effect of changing the 


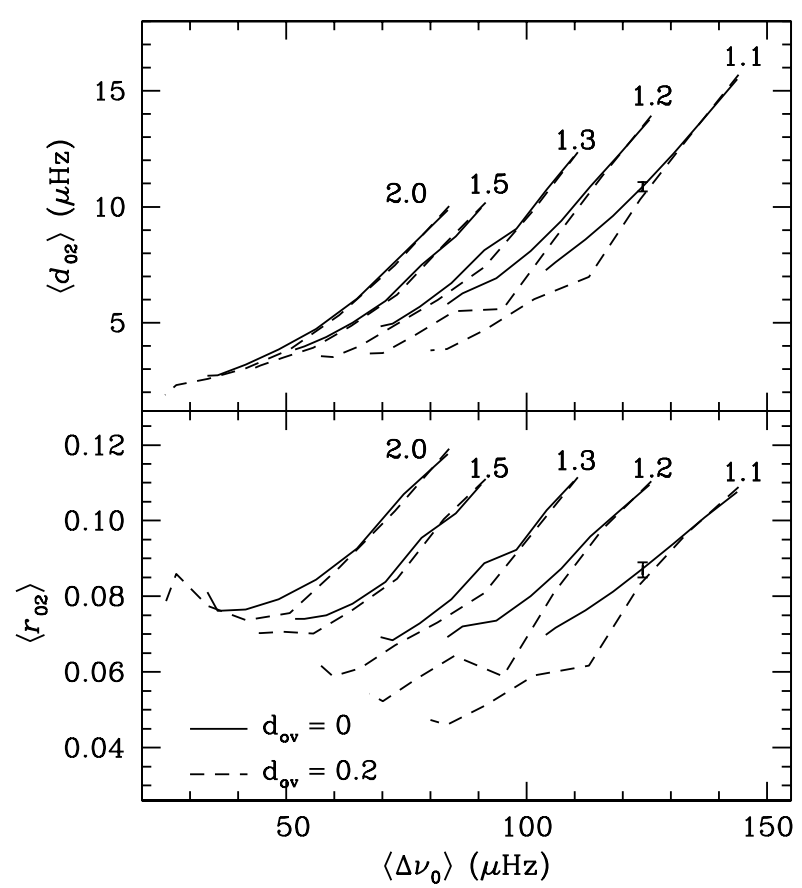

Fig. 2. The variation of the C-D diagram using the average small separations, $\left\langle d_{02}\right\rangle$ (top panel), and the average ratios, $\left\langle r_{02}\right\rangle$ (bottom panel) with convective core overshoot is shown. Two sets of models have been used - one with $d_{\mathrm{ov}}=0$ (solid curves) and another with $d_{\mathrm{ov}}=0.2$ (dashed curves) to illustrate the shift in the tracks. All other stellar parameters are identical in the two sets of models. Representative errorbars corresponding to relative frequency errors of $10^{-4}$ are shown.

mixing length reduces with mass. For low mass stars, the effect is comparable to the errors in $\left\langle d_{02}\right\rangle$ or $\left\langle r_{02}\right\rangle$, but the tracks are still close enough to allow an estimate of the stellar mass within $5 \%$.

The effect of convective core overshoot on the C-D diagram is more dramatic. We show this effect in Fig. 2 by comparing models without overshoot $\left(d_{\mathrm{ov}}=0\right)$ and those with $d_{\mathrm{ov}}=0.2$. The effect of overshoot in increasing the total radius of the star at a given evolutionary stage (in terms of the central hydrogen abundance, $X_{\mathrm{c}}$ ) is clearly seen in the extension of the tracks on the C-D diagram to lower values of $\left\langle\Delta v_{0}\right\rangle$. Further, the value of $\left\langle d_{02}\right\rangle$ also reduces with overshoot, reflecting the higher age of the star. In effect, the tracks on the C-D diagram deviate considerably from the standard ones when overshooting is included in the models. It is evident, therefore, that we need careful calibration of the effect of overshoot if one is to estimate the mass and age of a star (with mass $\gtrsim 1.1 M_{\odot}$ ) directly from the C-D diagram.

While the metallicity of a target star is often known from spectroscopic data, its helium content is typically unknown, thereby one is left with the ambiguity of one unknown parameter concerning the chemical composition. We consider three different scenarios which approximately reflect the various possibilities for the chemical composition of a star with a known metallicity (or $[\mathrm{Fe} / \mathrm{H}]$ ). We compare the tracks on the C-D diagram for all these cases with respect to those for the standard solar composition in Fig. 3.

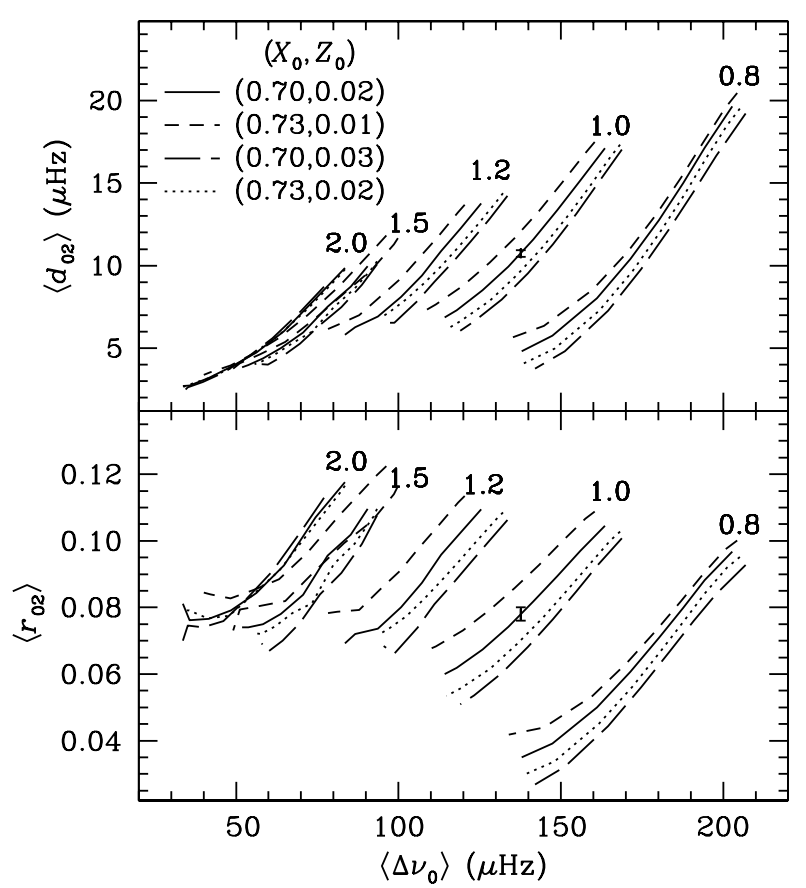

Fig. 3. The variation of the C-D diagram using the average small separations, $\left\langle d_{02}\right\rangle$ (top panel), and the average ratios, $\left\langle r_{02}\right\rangle$ (bottom panel) with initial chemical composition is shown. Four sets of models with different combinations of $\left(X_{0}, Z_{0}\right)$ have been used $-(0.70,0.02)$ (solid curves), $(0.73,0.01)$ (small dashed curves), $(0.70,0.03)$ (long dashed curves), and $(0.73,0.02)$ (dotted curves) to illustrate the shift in the tracks. All other stellar parameters are identical in the two sets of models. Representative errorbars corresponding to relative frequency errors of $10^{-4}$ are shown.

The first case $\left(\left(X_{0}, Z_{0}\right)=(0.73,0.01)\right)$ corresponds to a typical metal-poor star $([\mathrm{Fe} / \mathrm{H}] \sim-0.25)$ with proportionally reduced helium content. This would exemplify the situation where we would only have an indirect estimate of the helium abundance from the metallicity, based on assumptions about the chemical evolution of the galaxy. The second combination $\left(\left(X_{0}, Z_{0}\right)=(0.70,0.03)\right)$ mimics a star which is metal-rich $([\mathrm{Fe} / \mathrm{H}] \sim 0.25)$, but has near-solar helium abundance. The third case $\left(\left(X_{0}, Z_{0}\right)=(0.73,0.02)\right)$ reflects the chemical composition of a star which has solar metallicity, but is known to be over-abundant in hydrogen. It is evident that in each case, the tracks depend substantially on the chemical composition. It appears that even a slight change in the balance of helium and heavier elements at a constant hydrogen abundance produces a large shift in the C-D diagram. In summary, the effectiveness of the C-D diagram may be strongly undermined if the chemical composition is not fully known.

The importance of the equation of state (EOS) in the determination of internal stellar structure and its seismic manifestation cannot be ignored. However, by comparing two sets of models with OPAL and EFF (Eggleton et al. 1973) EOS we find that the shift in the tracks are at a level much lower than that induced by the other parameters discussed above, especially at higher masses. Therefore, it would be fair to deduce that the choice of the EOS would not greatly affect the extraction of the mass and age of a star from the C-D diagram. 


\section{3. $\Delta-t$ diagram}

\subsection{Probing sharp acoustic features}

While the C-D diagram is indeed a powerful tool to estimate the mass and age of a star from its oscillation frequencies, the process of averaging the frequency separations actually reduces the information content of the frequency spectrum. One of the other means to investigate the structure of the stellar interior from the oscillation frequencies without resorting to explicit modelling of the star is to utilise the oscillatory signal in the frequencies to determine the acoustic depth

$\tau_{\mathrm{d}}=\int_{r_{\mathrm{d}}}^{R} \frac{\mathrm{d} r}{c}$,

of a sharp feature, lying at a radius of $r_{\mathrm{d}}, c$ being the sound speed and $R$ the total radius of the star (e.g., Gough 1990).

The acoustic depth of the He II ionisation zone (hereafter referred to as HIZ) and the base of the convective envelope (hereafter referred to as BCZ) can be easily estimated using the second differences of the frequencies which are defined as $\delta^{2} v_{n, \ell}=v_{n+1, \ell}-2 v_{n, \ell}+v_{n-1, \ell}$ (Mazumdar \& Antia 2001). Following Basu et al. (2004), we fit a function of the form

$$
\begin{aligned}
\delta^{2} v= & \left(a_{1}+a_{2} v+\frac{a_{3}}{v^{2}}\right) \\
& +\left(b_{1}+\frac{b_{2}}{v}+\frac{b_{3}}{v^{2}}\right) \sin \left(4 \pi v \tilde{\tau}_{\mathrm{HIZ}}+\phi_{\mathrm{HIZ}}\right) \\
& +\left(c_{1}+\frac{c_{2}}{v}+\frac{c_{3}}{v^{2}}\right) \sin \left(4 \pi v \tilde{\tau}_{\mathrm{BCZ}}+\phi_{\mathrm{BCZ}}\right),
\end{aligned}
$$

to the second differences of the frequencies. The parameters $a_{i}, b_{i}, c_{i}(i=1,2,3)$ and $\tilde{\tau}_{\mathrm{HIZ}}, \tilde{\tau}_{\mathrm{BCZ}}, \phi_{\mathrm{HIZ}}$ and $\phi_{\mathrm{BCZ}}$ are obtained from a least-squares fit. The frequency range chosen for performing the fit is $1.5-3.0 \mathrm{mHz}$, after scaling by the factor $\left\langle\Delta v_{0}\right\rangle /\left\langle\Delta v_{0}\right\rangle_{\odot}$, where $\left\langle\Delta v_{0}\right\rangle$ and $\left\langle\Delta v_{0}\right\rangle_{\odot}(\simeq 135 \mu \mathrm{Hz})$ are the average large separations for the concerned star and the Sun respectively.

However, it is generally found that the acoustic depths of the BCZ and HIZ derived from the oscillatory signal in the frequencies $\left(\tilde{\tau}_{\mathrm{BCZ}}\right.$ and $\left.\tilde{\tau}_{\mathrm{HIZ}}\right)$ are systematically higher than their true values $\left(\tau_{\mathrm{BCZ}}\right.$ and $\left.\tau_{\mathrm{HIZ}}\right)$ calculated directly from the model according to the definition, Eq. (1). This is due to the fact that the estimation of the acoustic depth of the layer of discontinuity through the oscillatory signal in frequencies is biased by the surface phase shift (Vorontsov et al. 1991). However, as pointed out by Christensen-Dalsgaard et al. (1995) and Ballot et al. (2004), this bias can be largely eliminated in the estimation of the acoustic radii, $\tilde{t}_{\mathrm{BCZ}}$ and $\tilde{t}_{\mathrm{HIZ}}$ of the $\mathrm{BCZ}$ and HIZ, instead of the acoustic depths:

$\tilde{t}_{\mathrm{BCZ}}=\tilde{t}_{0}-\tilde{\tau}_{\mathrm{BCZ}}$, and $\tilde{t}_{\mathrm{HIZ}}=\tilde{t}_{0}-\tilde{\tau}_{\mathrm{HIZ}}$

where $\tilde{t}_{0} \equiv \frac{1}{2\left\langle\Delta v_{0}\right\rangle}$ is an estimate of the total acoustic radius of the star, $t_{0}=\int_{0}^{R} \mathrm{~d} r / c$.

By estimating the acoustic radii, $\tilde{t}_{\mathrm{BCZ}}$ and $\tilde{t}_{\mathrm{HIZ}}$, for several models of different mass and age by this method, we find that the true acoustic radii and the estimated values differ by $\$ 5 \%$ - a remnant effect of the surface phase shift. The differences tend to increase with mass, because for stars more massive than $\sim 1.5 M_{\odot}$ the outer convective envelope becomes thin and the BCZ and the HIZ lie close to each other. In such a situation, it is difficult to fit the two oscillatory components separately. Therefore, this method would not work very well for stars more massive than $1.5 M_{\odot}$.

This difference between the model values of the acoustic radii and their estimated values from the oscillatory signal exists even for exact frequencies and might be considered as a systematic bias in the process. In addition, there would be a certain random error in the case of actual frequency data associated with observational uncertainty. We have estimated this error through Monte Carlo simulations similar to that described in Sect. 2. We find that the uncertainty in the estimates of the mean density-scaled acoustic radii of the BCZ and the HIZ is typically around $40 \mathrm{~s}$ for relative frequency errors of $10^{-4}$. Since the uncertainty in the average large separation turns out to be tiny (Sect. 2), practically the entire contribution towards this error is from the fitting of $\tilde{\tau}_{\mathrm{BCZ}}$ and $\tilde{\tau}_{\mathrm{HIZ}}$. This is similar to the error margins provided by Basu et al. (2004), and comparable to that for the BCZ estimated by Ballot et al. (2004).

\subsection{A new seismic diagram}

We have seen in Sect. 2 that the average large separation, which is an index of the gross properties such as the mean density or the total acoustic size, can be determined to a fairly good accuracy from an observed frequency spectrum of a solar-type star. Further, in Sect. 3.1, we established that the acoustic position of the BCZ and the HIZ can be determined to an accuracy of at least $95 \%$ from the oscillatory signal in the frequencies despite the presence of some systematic bias due to unknown surface effects. Importantly, these estimates are obtained without explicit modelling of the stellar structure. However, the acoustic location of these layers inside the star cannot be independent of the general stratification or the chemical composition of the star. Therefore, the model-independent estimates of $\tilde{t}_{\mathrm{BCZ}}$ and $\tilde{t}_{\mathrm{HIZ}}$ indeed provide clues about the internal structure of the star.

In order to combine these independent pieces of information about the gross properties and the detailed structure of a star, we construct a diagram connecting the average large separation and the acoustic radius of the BCZ or the HIZ. The example of such a diagram, which we shall call the $\Delta-t$ diagram, is given in Fig. 4. Each line on this diagram is actually a locus of the acoustic radius of the BCZ (or the HIZ) as a function of the average large separation as a star of a given mass evolves on the main sequence. Thus it may be considered as a kind of seismic diagram, analogous to the C-D diagram. In this diagram, the ordinates are actually not the true acoustic radii $t_{\mathrm{BCZ}}$ and $t_{\mathrm{HIZ}}$, but rather are the values derived from the acoustic depths $\tau_{\mathrm{BCZ}}$ and $\tau_{\mathrm{HIZ}}$ (model values) and the mean large separation $\left\langle\Delta v_{0}\right\rangle$ (derived from exact frequencies), through Eq. (3). This is done deliberately to minimise the systematic error described in Sect. 3.1 in the calibration process. A diagram similar to that of Fig. 4 may be constructed using the acoustic radii $\tilde{t}_{\mathrm{HIZ}}$, instead of $\tilde{t}_{\mathrm{BCZ}}$. Since the properties of these diagrams are, 


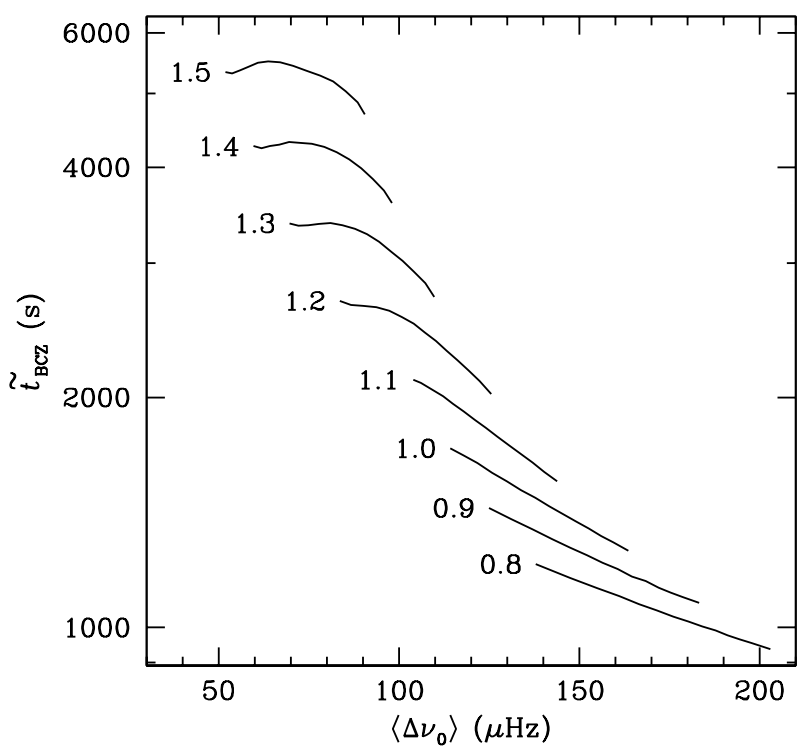

Fig. 4. An example of the $\Delta-t$ diagram, where the acoustic radius of the $\mathrm{BCZ}$ is plotted as a function of the average large separation. The ordinate is plotted in logarithmic values for the sake of clarity. Each curve on this diagram is an evolutionary track of the indicated mass in solar units.

in general, quite similar, it is enough to focus on the $\Delta-\tilde{t}_{\mathrm{BCZ}}$ diagram in the following discussion.

\subsection{Sensitivity of $\Delta-t$ diagram to stellar parameters}

It is evident from Fig. 4 that the $\Delta-t$ diagram has the diagnostic capability to predict the mass and evolutionary status of a star, in a manner similar to that of the C-D diagram. However, the tracks on this theoretical diagram would naturally depend on all the parameters involved in the stellar modelling. Direct determination of the mass and age would only be possible if the other stellar parameters are known a priori. Since typically this is not the case for real stars, we have investigated the sensitivity of the $\Delta-t$ diagram to stellar parameters by using several grids of models, for four different masses, 0.8, 1.0, 1.2 and $1.5 M_{\odot}$. Since the acoustic radii $\tilde{t}_{\mathrm{BCZ}}$ and $\tilde{t}_{\mathrm{HIZ}}$ cannot be reliably obtained for more massive stars, they are not relevant for this exercise.

In Fig. 5, we show the $\Delta-t$ tracks for models with two different values of the mixing length parameter, $\alpha=1.8$ and 1.6. The errorbars for $\tilde{t}_{\mathrm{BCZ}}$ for relative frequency errors of $10^{-4}$ are magnified by a factor of 3 in these diagrams to increase visibility. We find that the tracks for $\tilde{t}_{\mathrm{BCZ}}$ as a function of $\left\langle\Delta v_{0}\right\rangle$ are shifted almost parallelly as $\alpha$ is changed. The amount of this shift depends on the mass - while at lower masses it is too small to be detected given the uncertainty in the seismic determination of $\tilde{t}_{\mathrm{BCZ}}$, at masses $\gtrsim 1.2 M_{\odot}$, the shift is quite large. This would imply that this diagram is sensitive to $\alpha$ at higher masses, and might provide at least an indicative value of the same, provided other parameters are known.

The effect of convective core overshoot is expected to be small on these $\Delta-t$ diagrams, since the BCZ lies far apart from the convective core. Figure 6, however, confirms this only

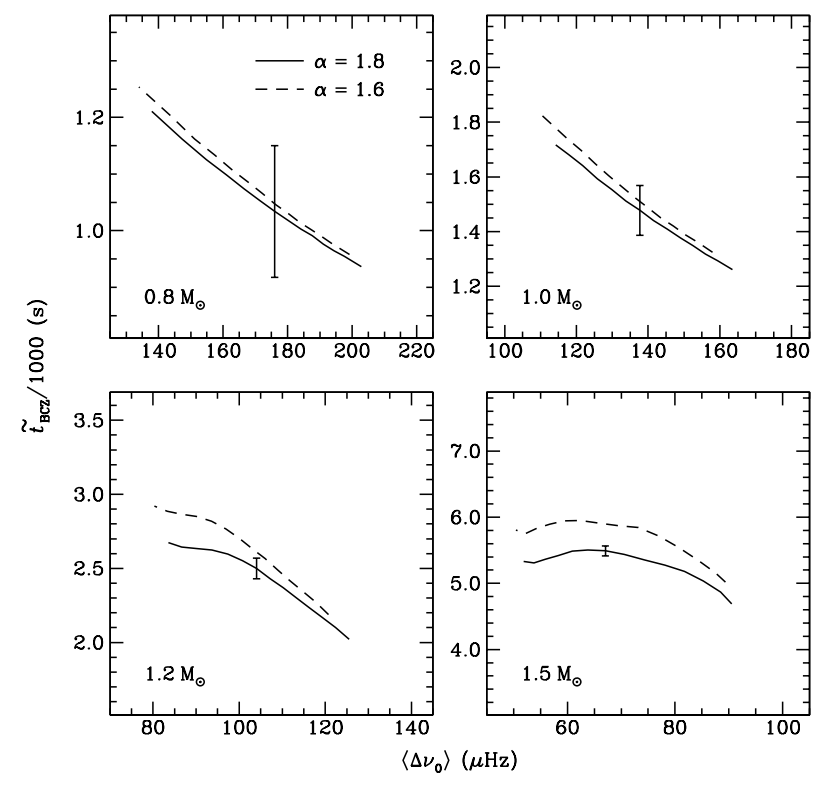

Fig. 5. The sensitivity of the $\Delta-t$ diagram with respect to the mixing length parameter $\alpha$ is illustrated. For each mass, indicated in the different panels, two sets of models have been used - one with $\alpha=1.8$ (solid curves) and another with $\alpha=1.6$ (dashed curves). All other stellar parameters remain identical. Representative $3 \sigma$ errorbars for the values of the acoustic radii for a relative error of $10^{-4}$ in frequencies are shown.

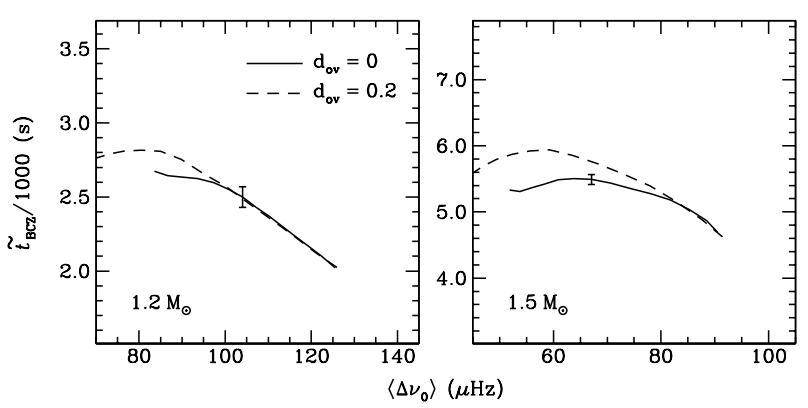

Fig. 6. The sensitivity of the $\Delta-t$ diagram to convective core overshooting is illustrated. Two sets of models have been used - one with $d_{\mathrm{ov}}=0$ (solid curves) and another with $d_{\mathrm{ov}}=0.2$ (dashed curves). All other stellar parameters remain identical. Representative $3 \sigma$ errorbars for the values of the acoustic radii for a relative error of $10^{-4}$ in frequencies are shown.

partially. We find that $\tilde{t}_{\mathrm{BCZ}}$ is indeed sensitive to $d_{\mathrm{ov}}$ at later stages of evolution for masses between 1.2 and $1.5 M_{\odot}$.

Figure 7 illustrates the effects of chemical composition on the $\Delta-t$ diagram. The compositions chosen for comparison to the standard one are identical to the ones described in Sect. 2. We find that the BCZ location is sensitive to the chemical content in all three scenarios. The effect is very pronounced at higher masses, which would mean that the $\Delta-t$ diagram may be used to constrain the chemical abundances, at least for $1.2-1.5 M_{\odot}$ stars. 


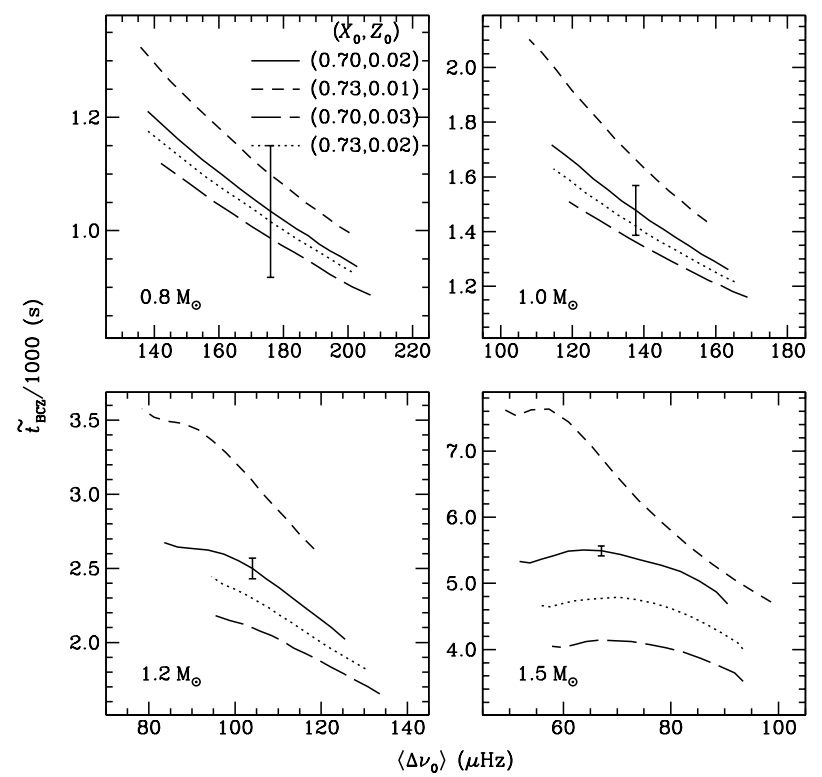

Fig. 7. The sensitivity of the $\Delta-t$ diagram to initial chemical composition is illustrated. Four sets of models with different combinations of $\left(X_{0}, Z_{0}\right)$ have been used $-(0.70,0.02)$ (solid curves), $(0.73,0.01)$ (small dashed curves), $(0.70,0.03)$ (long dashed curves), and $(0.73,0.02)$ (dotted curves). All other stellar parameters remain identical. Representative $3 \sigma$ errorbars for the values of the acoustic radii for a relative error of $10^{-4}$ in frequencies are shown.

\section{Application of the $\Delta-t$ diagram: the hare and hound exercise for HD 49933}

There might be two approaches to using the $\Delta-t$ diagram. The first is to determine any one parameter among $M,\left(X_{0}, Z_{0}\right), \alpha$ or $d_{\text {ov }}$ directly. But this would require independent knowledge about the remaining parameters, since each of these parameters affect the $\Delta-t$ diagram considerably. Some constraints about the mass, age and the chemical composition might be independently gathered in case the target star is a member of a binary system or a cluster. In such cases, it might be possible to use the $\Delta-t$ diagram to estimate the mixing length or the overshoot parameter.

However, we can also envisage the use of the $\Delta-t$ diagram in another way, when none of the stellar parameters are known a priori. In this case, given an initial trial model, one can test its relative position on the $\Delta-t$ diagram with respect to the observed data to gain insight into how a particular parameter needs to be changed from the initial guess in order to find a better match with the data. This process has to be iterative, but would considerably reduce the task of searching in a multidimensional parameter space for the best model.

In this section, we illustrate the use of the $\Delta-t$ diagram as applied successfully in the seismic modelling of a CoRoT primary target star, HD 49933, based on synthetic data in the course of a hare and hound exercise. This exercise, carried out within the CoRoT Seismology Working Group, was conducted in four principal steps - (i) construction of a theoretical model for a star, based on its position in the HR diagram and available information about its chemical composition, and calculation of its low-degree eigenfrequencies, (ii) conversion of the frequencies into a time series, taking into account the duration of CoRoT observation (150 days) and the expected instrumental and background stellar noise, (iii) extraction and mode identification of frequencies from this simulated time series, without knowledge of the original exact frequencies, and (iv) seismic interpretation of the extracted frequencies without knowledge of the original input model. Each of these steps were carried out by independent groups, maintaining confidentiality of the original input data, thereby simulating a situation which would be encountered for real data gathered from the CoRoT mission. The general description of this exercise can be found on the web (http://www.ias.u-psud.fr/virgo/html/corot/ datagroup/hh.html) and here we shall restrict ourselves only to the particular case of the seismic modelling of the star HD $49933^{1}$.

The basic parameters available for HD 49933 are summarised in Table 1 (stage 0). The data on which the seismic interpretation was based consisted of 67 individual frequencies of degree $\ell=0$ and 1, lying between 100 and $3100 \mu \mathrm{Hz}$, extracted in step (iii) described above. The errors in the frequencies ranged between 0.02 and $0.46 \mu \mathrm{Hz}$, with the highest errors reported for intermediate frequencies $(\sim 2000 \mu \mathrm{Hz})$. The average large separation, $\left\langle\Delta v_{0}\right\rangle$, was found to be $90.4 \pm 0.2 \mu \mathrm{Hz}$. Since the mean density of the star is not known at this stage, the average large separation was calculated for several different limits in the entire asymptotic range, and the variation in its value was included as an additional error. The mean density was estimated to be $0.465 \pm 0.015 \bar{\rho}_{\odot}$ through a calibration of the average large separation. Unfortunately, only a few frequencies for $\ell=2$ and 3 could be extracted from the simulated time series, and therefore, we had no small separations $d_{02}$ to utilise in our seismic analysis. This automatically ruled out the use of the C-D diagram.

We derived the acoustic depths $\tilde{\tau}_{\mathrm{BCZ}}$ and $\tilde{\tau}_{\mathrm{HIZ}}$ by fitting an expression, similar to that of Eq. (2) to the second differences of the frequencies, as shown in Fig. 8. For this exercise, we used all modes between 700 and $2700 \mu \mathrm{Hz}$, the range being chosen to use as many modes as possible, while not deviating too much from the standard choice as explained by Basu et al. (2004). This yielded values of $\tilde{t}_{\mathrm{BCZ}}=4085 \pm 68 \mathrm{~s}$ and $\tilde{t}_{\mathrm{HIZ}}=4866 \pm 47 \mathrm{~s}$. The comparison of these results to the original model values are shown in Table 1 (stage 1). The excellent agreement of these values, obtained prior to any modelling of the star, indeed confirms the diagnostic power of the oscillatory signal in the frequencies. In practice, however, the original values were not revealed at this stage, but only at the end of the entire exercise.

In the next step, we proceeded to use the $\Delta-t$ diagrams to arrive at the closest model for the star, already taking into account the constraints imposed on the stellar parameters by the position of HD 49933 on the HR diagram. In the top panel of Fig. 9, we show the evolutionary tracks of three sets of models

1 The relevant work in steps (i), (ii) and (iii) for HD 49933 was carried out by I. Roxburgh, C. Barban and T. Toutain respectively. The seismic interpretation in step (iv) described here was carried out by the author in collaboration with E. Michel. 
Table 1. The input parameters and results from a seismic analysis of HD 49933 in a mock exercise are shown. The table is divided in three parts, classified by stages of the seismic analysis: (0) initial constraints provided to both modelling team and seismic interpretation team; (1) results obtained from seismic analysis, prior to modelling; (2) final results obtained from seismic modelling.

\begin{tabular}{ccccc}
\hline \hline Stage & Quantity & $\begin{array}{c}\text { Non-Seismic } \\
\text { constraint }\end{array}$ & $\begin{array}{c}\text { Seismic } \\
\text { value }\end{array}$ & $\begin{array}{c}\text { Original } \\
\text { input }\end{array}$ \\
\hline 0 & $T_{\text {eff }}$ & $6700 \pm 100 \mathrm{~K}$ & & \\
& $M_{\text {bol }}$ & $3.35 \pm 0.10 \mathrm{mag}$ & & \\
& {$[\mathrm{Fe} / \mathrm{H}]$} & $-0.32 \pm 0.10$ & & \\
\hline 1 & $\bar{\rho} / \bar{\rho}_{\odot}$ & & $0.465 \pm 0.015$ & 0.467 \\
& $\tilde{t}_{\mathrm{BCZ}}$ & $4085 \pm 68 \mathrm{~s}$ & $4031 \mathrm{~s}$ \\
& $\tilde{t}_{\mathrm{HIZ}}$ & & $4866 \pm 49 \mathrm{~s}$ & $4805 \mathrm{~s}$ \\
\hline 2 & $M / M_{\odot}$ & & $1.24 \pm 0.01$ & 1.25 \\
& $X_{\mathrm{c}}$ & & $0.495 \pm 0.010$ & 0.50 \\
& $X_{0}$ & & $0.72 \pm 0.01$ & 0.73 \\
& $Z_{0}$ & & $0.01 \pm 0.005$ & 0.01 \\
& $d_{\mathrm{ov}}$ & & $0.20 \pm 0.01$ & 0.20 \\
& $\alpha$ & & $1.60 \pm 0.05$ & 1.50 \\
\hline
\end{tabular}

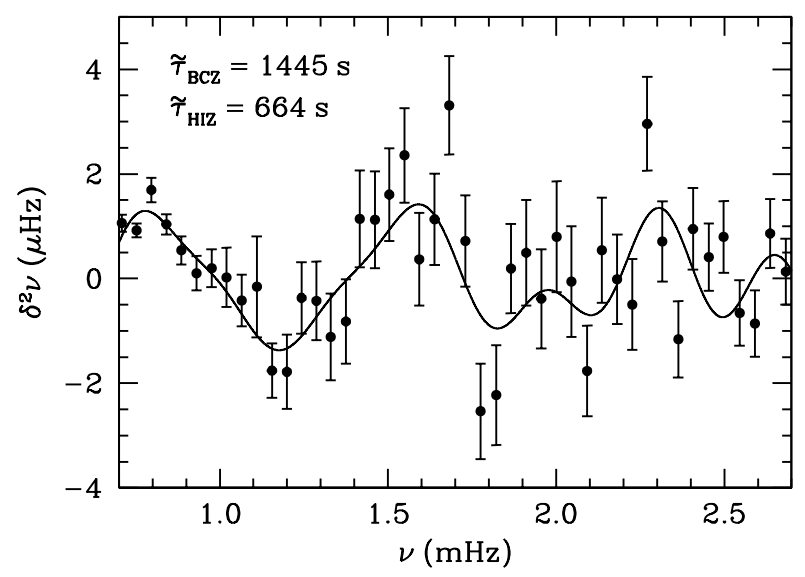

Fig. 8. The oscillatory signal in the second differences of the frequencies of HD 49933 (shown as data points with respective errorbars) are fitted by the expression (2) (solid curve) to extract the values of $\tilde{\tau}_{\mathrm{BCZ}}$ and $\tilde{\tau}_{\mathrm{HIZ}}$.

with the same mass, overshoot and the mixing length parameter, but differing in chemical composition, and passing through the errorbox of HD 49933. The $\Delta-t$ diagram is illustrated in the bottom panel, where the range in the values of $\left\langle\Delta v_{0}\right\rangle$ and $\tilde{t}_{\mathrm{BCZ}}$ for each track are restricted to correspond to their overlap with the box on the HR diagram. The box on the $\Delta-t$ diagram represents the values of $\left\langle\Delta v_{0}\right\rangle$ and $\tilde{t}_{\mathrm{BCZ}}$ that we have already derived from the frequencies. This is an example of one of the $\Delta-t$ diagrams that we utilised in this analysis, in each case varying one stellar parameter.

The significant result to be noted here is that a much stronger constraint on the varying parameter (in this case the chemical composition) and the age can be obtained by
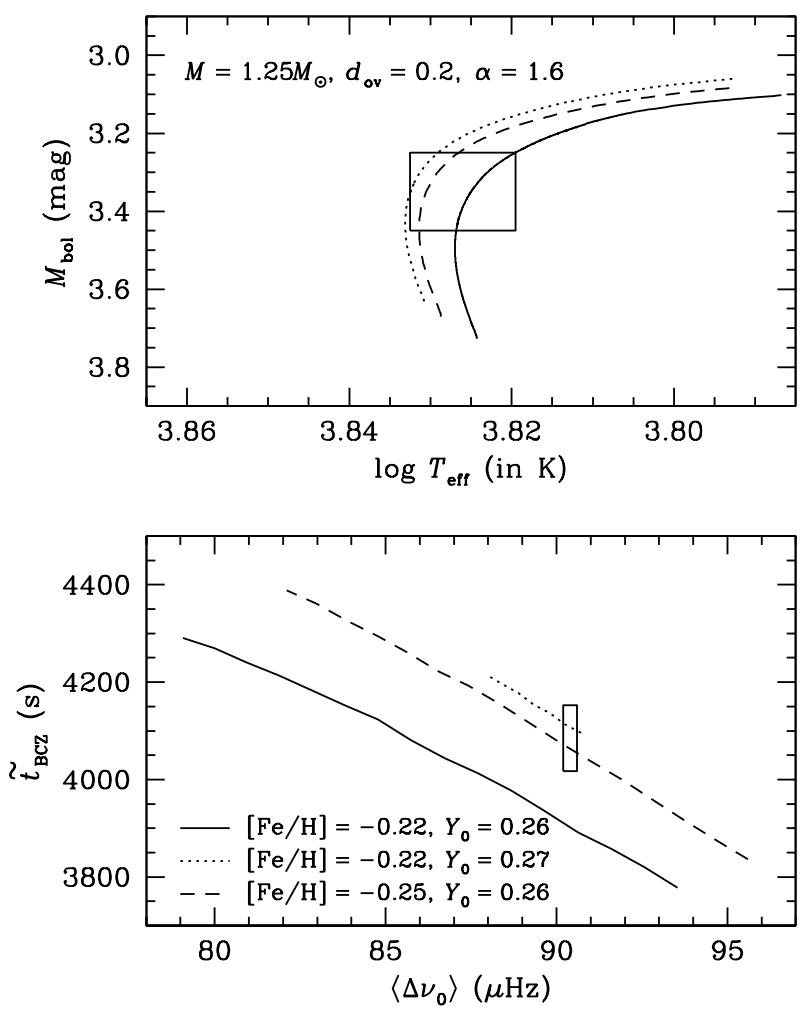

Fig. 9. Top panel: the position of HD 49933 is shown on the HR diagram. Three evolutionary tracks for models with a given mass, overshoot and mixing length parameter, but different chemical compositions are illustrated. Bottom panel: the $\Delta-t$ diagram for $\tilde{t}_{\mathrm{BCZ}}$ is shown with the three tracks only for the portion where they lie inside the box in the top panel. The derived values of $\left\langle\Delta v_{0}\right\rangle$ and $\tilde{t}_{\mathrm{BCZ}}$ for HD 49933 are represented by a box.

invoking the $\Delta-t$ diagram than is possible through the errorbox on the HR diagram alone. Of course, this is not the definitive set of parameters for the best possible model, since we have allowed only the chemical composition to vary, while fixing the other parameters. We need to change all the parameters one by one within the range allowed by the HR diagram, and test for the remaining parameter through the $\Delta-t$ diagram. But the strength of the $\Delta-t$ diagram lies in the fact that starting from an initial guess model, we can get a very good indication as to in which direction we should move in the multi-dimensional parameter space to arrive at the best model. The task of searching for the best possible combination of parameters is greatly reduced by the $\Delta-t$ diagram through the additional information about the location of the BCZ and HIZ.

After several iterations, we were able to converge to a set of models that satisfied the constraints on the $\Delta-t$ diagram for both $\tilde{t}_{\mathrm{BCZ}}$ and $\tilde{t}_{\mathrm{HIZ}}$. The final best models were selected on the basis of comparisons of large separation values between the data and the models. The absence of small separations in the data prevented us from constraining the parameters further. We estimated errors on each parameter to represent the range of values that would produce models which are all consistent with the errorbox on the $\Delta-t$ diagram, as well as the individual large separation values. Needless to add, these models are already consistent with the limits imposed on the HR diagram. 
The parameters for our best models are shown in Table 1 (stage 2), alongwith those used for the original input model. Clearly, a remarkably good match with the input model was achieved. We should add a caveat though - as it turned out, both the input modelling and the seismic analysis had utilised the same evolutionary code, CESAM; this might have contributed partially to the exactness of the result. However, the important point is not the goodness of the fit, but rather the illustration of the use of a new seismic technique, the $\Delta-t$ diagram, which led us to the final result.

\section{Conclusions}

We have investigated the possibility of using the C-D diagram for real asteroseismic data by comparing the error in the separations propagated from the observed frequencies against the sensitivity of the tracks toward different stellar parameters. The largest uncertainty in calibrating the C-D diagram arises from the convective core overshoot parameter and the chemical composition of the star. However, the effect of the mixing length parameter and the equation of state are found to be relatively small. We estimate that if the chemical composition is known independently, it would be possible to determine the mass and age of a low mass star within 5\% using the average frequency separations. For a star with a convective core, the uncertainty would increase due to the unknown extent of convective core overshoot.

We propose a new kind of seismic diagram (which we call the $\Delta-t$ diagram) for low mass main sequence stars, connecting the acoustic location of sharp features inside a star and its mean large separation. Since the acoustic location of the base of the convective envelope or the He II ionisation zone can be estimated to a precision of $\sim 95 \%$, the position of a star on this diagram can be determined fairly accurately. The relation of such sharp features with the internal stratification of the star and the dependence of the mean large separation on its gross properties constitutes the physical basis behind this diagram. This diagram is designed to help in modelling the star by exploiting the sensitivity of the acoustic location of the sharp features toward different stellar parameters.

The use of the $\Delta-t$ diagram is illustrated through the seismic interpretation of simulated data for a CoRoT primary target star, HD 49933, carried out in the form of a hare and hound exercise. Firstly, independent of comparison of actual models, the acoustic radii of the base of the convective envelope and the He II ionisation zone are recovered to a good accuracy by analysing the oscillatory signal in the second differences of the frequencies. Further, all the stellar parameters used in the original input model could be correctly estimated through the seismic modelling based on the $\Delta-t$ diagram.

It is found that the $\Delta-t$ diagram can constrain the multidimensional space of stellar parameters substantially and provide an iterative method of quickly converging to the best model, starting from an initial guess. The success of the method could be somewhat sensitive to the position of the target star on the HR diagram, since the effect of the various stellar parameters on the $\Delta-t$ diagram is not uniform with respect to mass or age of the star. We find that at least in the mass range of $\sim 1-1.5 M_{\odot}$, this method is likely to be powerful. The efficiency can be further enhanced if the $\Delta-t$ diagram can be used in conjunction with the C-D diagram, which was unfortunately not possible in this particular hare and hound exercise.

The application of asteroseismic diagrams to oscillation frequencies of solar-type stars looks to be quite plausible, provided the dependence of these diagrams on different unknown stellar parameters is taken into account in the calibration process. On the other hand, this sensitivity of seismic diagrams like the $\Delta-t$ diagram can actually be exploited to construct reliable models for target stars based on the model-independent results obtained from their oscillation frequencies. Clearly, more tests are required with real data to refine these methods.

Acknowledgements. I would like to thank Ian Roxburgh, Caroline Barban, Thierry Toutain and Eric Michel for their contribution to the results of the CoRoT Hare \& Hound exercise presented here. This work was supported partially by CEFIPRA (Centre Franco-Indien pour la Promotion de la Recherche Avancée) project No. 2504-3, and by the Research Fund, KU Leuven under the grant GOA/2003/04.

\section{References}

Alexander, D. R., \& Ferguson J. W. 1994, ApJ, 437, 879

Angulo, C., Arnould, M., \& Rayet, M. (NACRE collaboration) 1999, Nucl. Phys. A, 656, 1

Audard, N., Provost, J., \& Christensen-Dalsgaard, J. 1995, A\&A, 297, 427

Baglin, A. 2003, AdSpR, 31, 345

Ballot, J., Turck-Chièze, S., \& García, R. A. 2004, A\&A, 423, 1051

Basu, S., Antia, H. M., \& Narasimha, D. 1994, MNRAS, 267, 209

Basu, S., Mazumdar, A., Antia, H. M., \& Demarque, P. 2004, MNRAS, 350, 277

Bedding, T. R., \& Kjeldsen, H. 2003, PASA, 20, 203

Christensen-Dalsgaard, J. 1988, in Advances in Helio and Asteroseismology, ed. J. Christensen-Dalsgaard, \& S. Frandsen (Reidel), 295

Christensen-Dalsgaard, J. 2004, Sol. Phys., 220, 137

Christensen-Dalsgaard, J., \& Berthomieu, G. 1991, Solar interior and atmosphere (University of Arizona Press), 401

Christensen-Dalsgaard, J., Monteiro, M. J. P. F. G., \& Thompson, M. J. 1995, MNRAS, 276, 283

Eggleton, P. P., Faulkner, J., \& Flannery, B. P. 1973, A\&A, 23, 325

Floranes, H. O., Christensen-Dalsgaard, J., \& Thompson, M. J. 2005, MNRAS, 356, 671

Gough, D. O. 1990, in Lecture Notes in Physics 367, ed. Y. Osaki, \& H. Shibahashi (Berlin: Springer), 283

Henyey, L., Vardya, M. S., \& Bodenheimer, P. 1965, ApJ, 142, 841

Iglesias, C. A., \& Rogers, F. J. 1996, ApJ, 464, 943

Mazumdar, A., \& Antia, H. M. 2001, A\&A, 377, 192

Mazumdar, A., \& Roxburgh, I. 2003, Asteroseismology Across the HR Diagram, 477

Monteiro, M. J. P. F. G., Christensen-Dalsgaard, J., \& Thompson, M. J. 1994, A\&A, 283, 247

Morel, P. 1997, A\&AS, 124, 597

Rogers, F. J., \& Nayfonov, A. 2002, ApJ, 576, 1064

Roxburgh, I. W., \& Vorontsov, S. V. 1994, MNRAS, 268, 880

Roxburgh, I. W., \& Vorontsov, S. V. 2003, A\&A, 411, 215

Walker, G., Matthews, J., Kuschnig, R., et al. 2003, PASP, 115, 1023

Vorontsov, S. V., Baturin, V. A., \& Pamiatnykh, A. A. 1991, Nature, 349,49 
A. Mazumdar: Asteroseismic diagrams for solar-type stars, Online Material $p 1$

\section{Online Material}




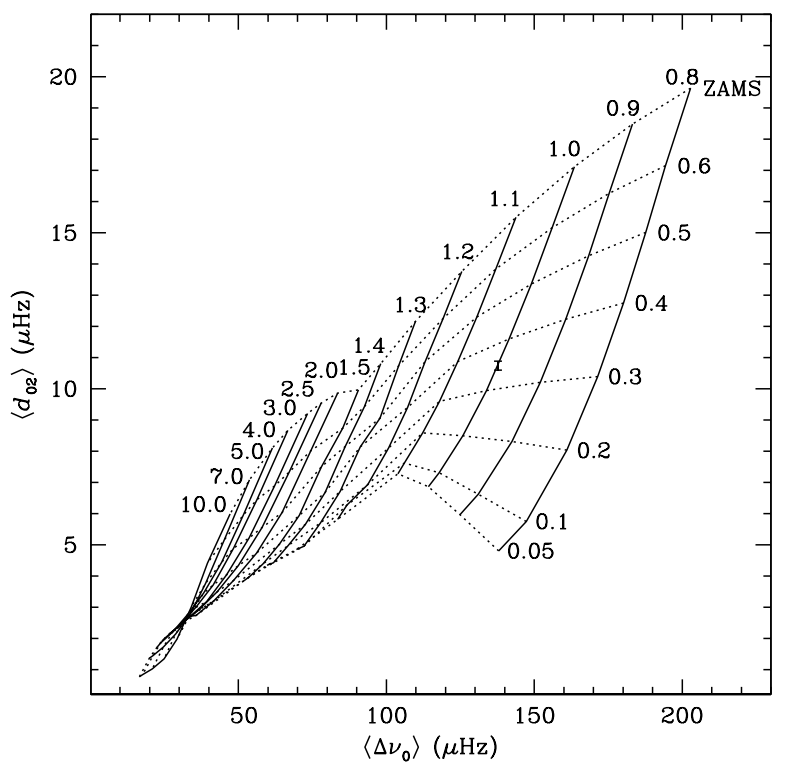

Fig. 10. The classical C-D diagram, plotting the average small separation, $\left\langle d_{02}\right\rangle$, against the average large separation, $\left\langle\Delta v_{0}\right\rangle$, for a grid of main sequence models between mass $0.8 M_{\odot}$ and $10 M_{\odot}$. The bold lines follow the evolution of each stellar model with a given mass, which is indicated at the top of the track. The dotted lines connect the models at same stages of evolution, indicated by the central hydrogen abundance, $X_{\mathrm{c}}$. Representative errorbar for $\left\langle d_{02}\right\rangle$ of a $1 M_{\odot}$ star, corresponding to relative frequency errors of $10^{-4}$ is shown.

\section{The classical C-D Diagram and its variations}

We present a "classical" C-D diagram in Fig. 10, involving the large separations $\left\langle\Delta v_{0}\right\rangle$, and the small separations $\left\langle d_{02}\right\rangle$. The stellar models used for this diagram were constructed using the OPAL equation of state and had an initial chemical composition of $\left(X_{0}, Z_{0}\right)=(0.70,0.02)$. The mixing length parameter (in terms of the local pressure scale height), $\alpha$, is 1.8 and no convective core overshoot was considered.

In Fig. 11, the corresponding C-D diagram using the ratios of separations is shown. As pointed out by ChristensenDalsgaard (2004), the filtering out of the surface effects indeed increases the orthogonality of the two effects regarding mass and age. Further, we find that this C-D diagram has improved "resolution" at higher masses, i.e., the lines corresponding to masses greater than $2 M_{\odot}$ do not collapse as much as in the classical C-D diagram (cf. Fig. 10). This implies that the diagnostic capability of the C-D diagram can both be improved and extended to higher masses if we use the ratios of separations instead of the separations themselves.

The kinks seen in the tracks for models with mass 1.2-1.5 $M_{\odot}$ are not results of inconsistencies in the models, but are in fact related to the evolution of the convective core. In this mass range, the convective core grows in mass (or radius) for a significant part of the main sequence evolution, before beginning to shrink later. The kink corresponds to the evolutionary phase where this phase of growth of the convective core ends and the shrinking phase begins. This effect is even more pronounced in models including convective core overshoot (see Fig. 2).

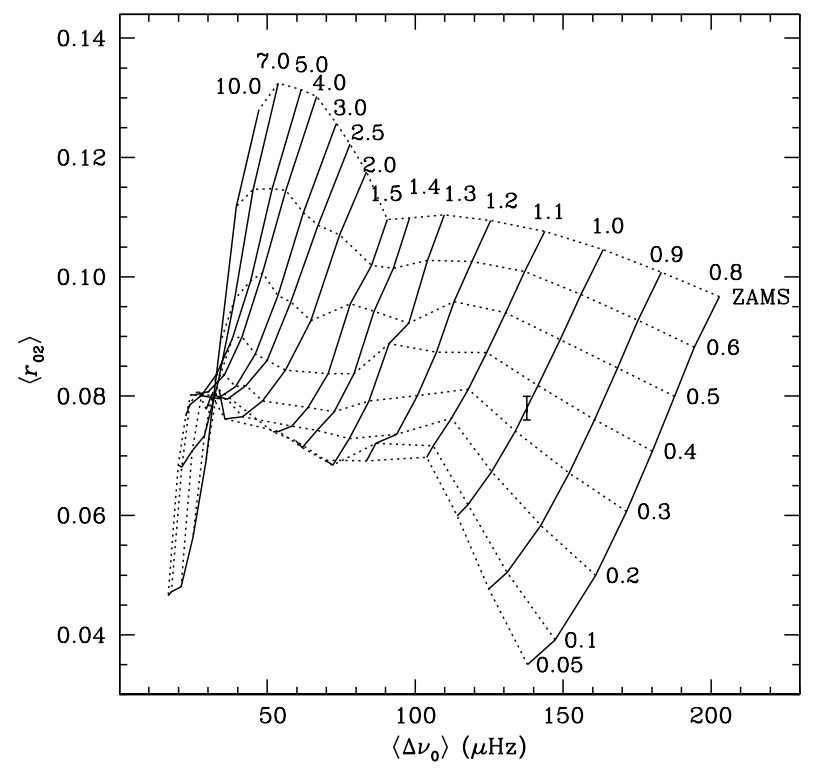

Fig. 11. A C-D diagram using the average ratio, $\left\langle r_{02}\right\rangle$, of the small separation to the large separation. The stellar models used are identical to those in Fig. 10.

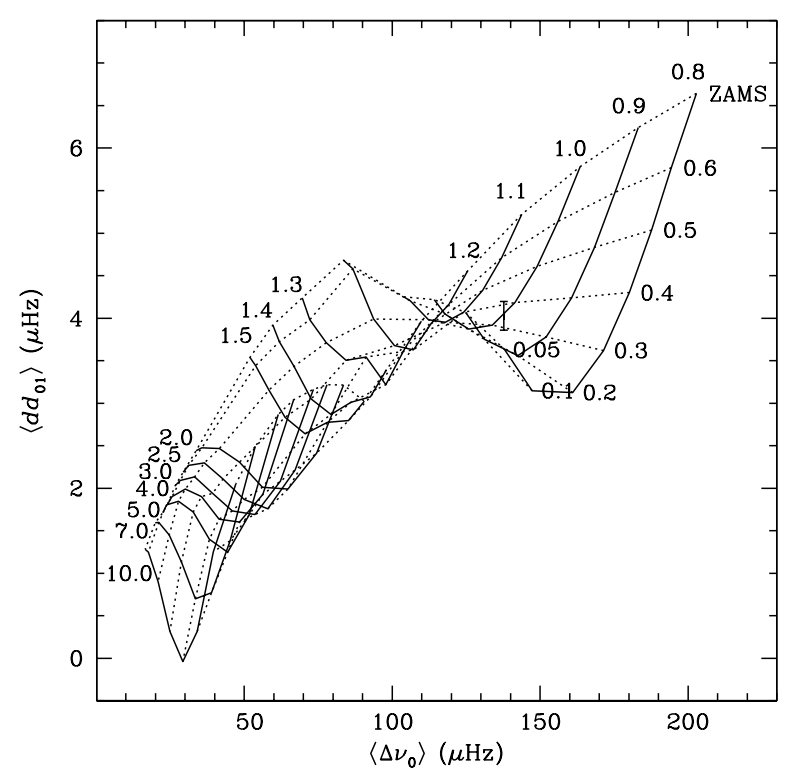

Fig. 12. An alternative C-D diagram using the average small separations $\left\langle d d_{01}\right\rangle$. The stellar models used are identical to those in Fig. 10.

The construction of the C-D diagram illustrated above requires the determination of a set of $\ell=0$ mode frequencies as well as $\ell=2$ frequencies of similar radial orders. In practice, however, we might encounter the situation where only $\ell=0$ and $\ell=1$ modes are identified for a target star, but not the $\ell=2$ modes. Following Eqs. (4) and (5) of Roxburgh \& Vorontsov (2003), we can define the 5-point separations between these two sets of modes. The C-D diagrams with these separations and the corresponding ratios (Eq. (8) of the same paper) are shown in Figs. 12 and 13. 
A. Mazumdar: Asteroseismic diagrams for solar-type stars, Online Material p 3

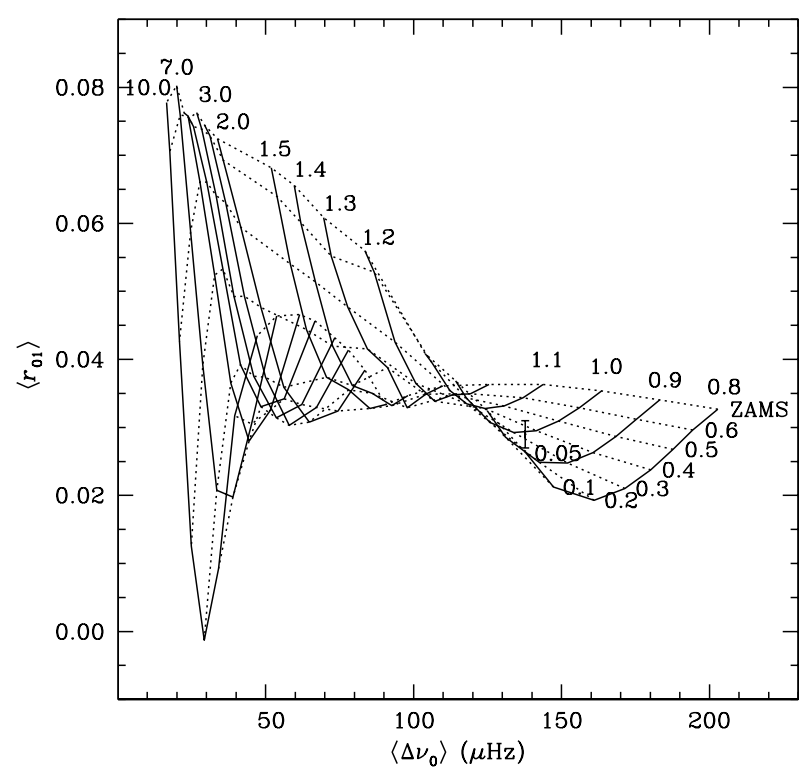

Fig. 13. A C-D diagram using the average ratio, $\left\langle r_{01}\right\rangle$, of the small separation to the large separation. The stellar models used are identical to those in Fig. 10.

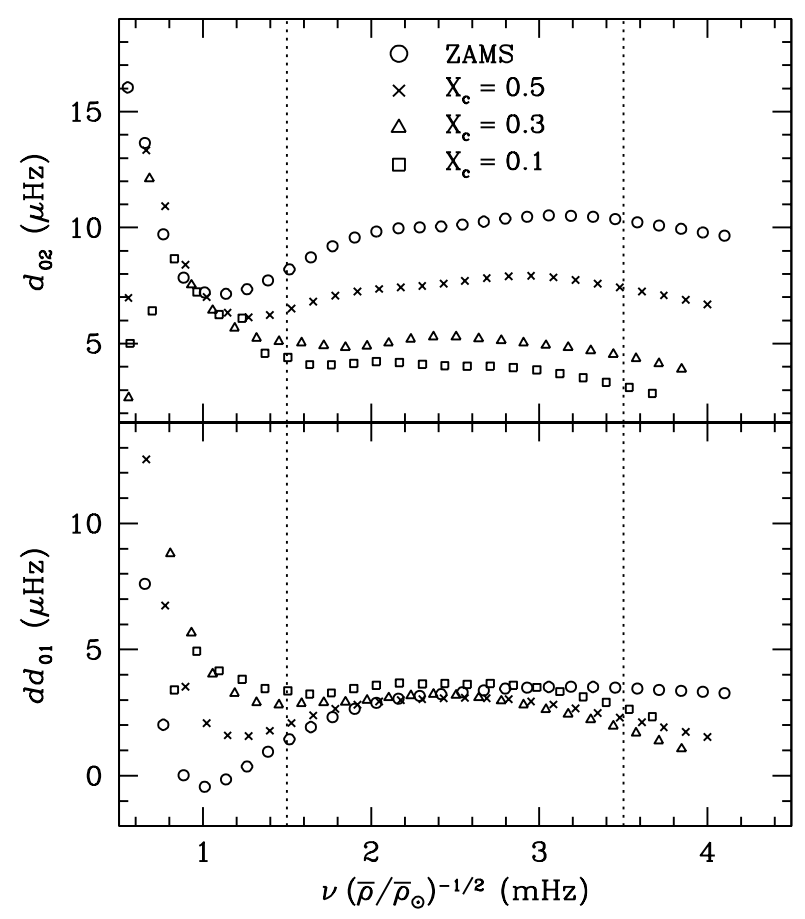

Fig. 14. The small separations $d_{02}$ (top panel) and $d d_{01}$ (bottom panel) are shown as functions of the scaled frequency for four $1.5 M_{\odot}$ models at different stages of evolution. The limits of scaled frequency used to compute the averages for the C-D diagrams are indicated by the dotted lines.

It is interesting to note the difference in the structure of the two diagrams in Figs. 10 and 12. The C-D diagram with the $\left\langle d d_{01}\right\rangle$ separations undergoes a "twist" at higher masses. This is, in fact, to be expected from the behaviour of these separations as functions of frequency. In Fig. 14 we plot both $d_{02}$ and $d d_{01}$ as functions of the scaled frequency, $v / \sqrt{\bar{\rho} / \bar{\rho}_{\odot}}$, where $\bar{\rho}$ denotes the mean density, for a sequence of models of mass $1.5 M_{\odot}$ at different evolutionary stages on the main sequence. The range of scaled frequencies used to compute the average values in Figs. 10 and 12 are shown as well. We find that while the value of $d_{02}$ decreases monotonically with age at all frequencies, at low frequencies the value of $d d_{01}$ actually increases with age, flattening out to an almost constant value at intermediate frequencies. At frequencies close to the acoustic limit $d d_{01}$ decreases slightly with age. This explains the "twist" in Fig. 12. Evidently, the range of frequencies over which the average values are computed plays a crucial role in determining the structure of the C-D diagram. The physical reason for this difference of behaviour between $d_{02}$ and $d d_{01}$, as hinted by Audard et al. (1995), possibly lies in the difference in the position of the inner turning points of the $\ell=1$ and $\ell=2$ modes close to the stellar core. While the lowest frequency $\ell=1$ modes would be reflecting off a layer close to the peak in the Brunt-Väisälä frequency at the boundary of the convective core, the $\ell=2$ modes may not be penetrating that far. The difference in behaviour of these two small separations bears a clue to the evolution of the Brunt-Väisälä frequency with age. 